\title{
DETECTION OF VHE $\gamma$-RAYS FROM HESS J0632+057 DURING THE 2011 FEBRUARY X-RAY OUTBURST WITH THE MAGIC TELESCOPES
}

J. Aleksić ${ }^{1}$, E. A. Alvarez ${ }^{2}$, L. A. Antonelli ${ }^{3}$, P. Antoranz ${ }^{4}$, M. Asensio ${ }^{2}$, M. Backes ${ }^{5}$, U. Barres de Almeida ${ }^{6}$, J. A. Barrio ${ }^{2}$, D. Bastieri ${ }^{7}$, J. Becerra González ${ }^{8}$, W. BednareK ${ }^{9}$, K. Berger $^{8,10}$, E. Bernardini ${ }^{11}$, A. Biland ${ }^{12}$, O. Blanch ${ }^{1}$, R. K. Bock ${ }^{6}$, A. Boller ${ }^{12}$, G. Bonnoli ${ }^{3}$, D. Borla Tridon ${ }^{6}$, V. Bosch-Ramon ${ }^{13}$, T. Bretz ${ }^{14,27}$, A. Cañellas ${ }^{13}$, E. Carmona ${ }^{6,28}$, A. Carosi ${ }^{3}$, P. Colin ${ }^{6}$, E. Colombo ${ }^{8}$, J. L. Contreras ${ }^{2}$, J. Cortina ${ }^{1}$, L. Cossio ${ }^{15}$, S. Covino ${ }^{3}$, P. Da Vela ${ }^{4}$, F. Dazzi ${ }^{15,29}$, A. De Angelis ${ }^{15}$, G. De Caneva ${ }^{11}$, E. De Cea del Pozo ${ }^{16}$, B. De Lotto ${ }^{15}$, C. Delgado Mendez ${ }^{8,29}$,

A. Diago Ortega ${ }^{8,10}$, M. Doert ${ }^{5}$, A. Domínguez ${ }^{17}$, D. Dominis Prester ${ }^{18}$, D. Dorner $^{12}$, M. Doro ${ }^{19}$, D. Eisenacher ${ }^{14}$, D. Elsaesser ${ }^{14}$, D. Ferenc ${ }^{18}$, M. V. Fonseca ${ }^{2}$, L. FonT ${ }^{19}$, C. FrucK ${ }^{6}$, R. J. García LóPez ${ }^{8,10}$, M. GarCZarczyK ${ }^{8}$, D. Garrido Terrats ${ }^{19}$, G. Giavitto ${ }^{1}$, N. Godinović ${ }^{18}$, A. González Muñoz ${ }^{1}$, S. R. Gozzini ${ }^{11}$, D. Hadasch ${ }^{16}$, D. HäFner ${ }^{6}$, A. Herrero ${ }^{8,10}$, D. Hildebrand ${ }^{12}$, J. Hose $^{6}$, D. Hrupec ${ }^{18}$, B. Huber ${ }^{12}$, F. Jankowski ${ }^{11}$, T. Jogler ${ }^{6,30}$, V. Kadenius ${ }^{20}$, H. KellermanN ${ }^{6}$, S. Klepser ${ }^{1}$, T. KräHenbüHL ${ }^{12}$, J. Krause ${ }^{6}$, A. La Barbera ${ }^{3}$, D. Lelas ${ }^{18}$, E. Leonardo ${ }^{4}$, N. LeWANDOWSKA ${ }^{14}$, E. LindFors ${ }^{20}$, S. LOMBARDI ${ }^{7}$, M. LóPEZ ${ }^{2}$, R. LóPEZ-Coto ${ }^{1}$, A. LóPEZ-Oramas ${ }^{1}$, E. LORENZ ${ }^{6,12}$, M. Makariev ${ }^{21}$, G. Maneva ${ }^{21}$, N. MankuZhiyiL ${ }^{15}$, K. Mannheim ${ }^{14}$, L. Maraschi ${ }^{3}$, M. Mariotti ${ }^{7}$, M. MartíneZ ${ }^{1}$, D. Mazin ${ }^{1,6}$, M. Meucci ${ }^{4}$, J. M. Miranda ${ }^{4}$, R. Mirzoyan ${ }^{6}$, J. MoldóN ${ }^{13}$, A. Moralejo ${ }^{1}$, P. Munar-Adrover ${ }^{13}$,

A. Niedzwiecki ${ }^{9}$, D. Nieto ${ }^{2}$, K. Nilssson ${ }^{20,31}$, N. NowaK ${ }^{6}$, R. Orito ${ }^{22}$, S. Paiano ${ }^{7}$, D. Paneque ${ }^{6}$, R. Paoletti ${ }^{4}$, S. Pardo $^{2}$, J. M. Paredes ${ }^{13}$, S. Partini ${ }^{4}$, M. A. Perez-Torres ${ }^{1}$, M. Persic ${ }^{15,23}$, M. Pilia ${ }^{24}$, J. Pochon ${ }^{8}$, F. Prada ${ }^{17}$, P. G. Prada Moroni ${ }^{25}$, E. Prandini ${ }^{7}$, I. Puerto Gimenez ${ }^{8}$, I. PuljaK ${ }^{18}$, I. Reichardt ${ }^{1}$, R. Reinthal ${ }^{20}$, W. Rhode ${ }^{5}$, M. Ribó ${ }^{13}$, J. Rico ${ }^{1,26}$,

S. RÜGamer ${ }^{14}$, A. Saggion ${ }^{7}$, K. Saito ${ }^{6}$, T. Y. Saito ${ }^{6}$, M. Salvati ${ }^{3}$, K. Satalecka ${ }^{2}$, V. Scalzotto ${ }^{7}$, V. Scapin ${ }^{2}$, C. Schultz $^{7}$,

T. Schweizer ${ }^{6}$, S. N. Shore ${ }^{25}$, A. Sillanpä ${ }^{20}$, J. Sitarek ${ }^{1,9}$, I. SNidaric ${ }^{18}$, D. Sobczynska ${ }^{9}$, F. Spanier ${ }^{14}$, S. Spiro ${ }^{3}$, V. Stamatescu ${ }^{1}$, A. Stamerra ${ }^{4}$, B. Steinke ${ }^{6}$, J. Storz ${ }^{14}$, N. Strah ${ }^{5}$, S. Sun ${ }^{6}$, T. Surić ${ }^{18}$, L. TaKalo $^{20}$, H. Takami $^{6}$, F. Tavecchio ${ }^{3}$, P. Temnikov ${ }^{21}$, T. Terzić ${ }^{18}$, D. Tescaro ${ }^{8}$, M. Teshima $^{6}$, O. Tibolla ${ }^{14}$, D. F. Torres ${ }^{16,26}$, A. Treves $^{24}$,

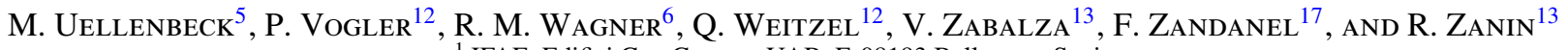
${ }^{1}$ IFAE, Edifici Cn., Campus UAB, E-08193 Bellaterra, Spain

${ }^{2}$ Grupo de Fisica Altas Energias, Universidad Complutense, E-28040 Madrid, Spain ${ }^{3}$ INAF National Institute for Astrophysics, I-00136 Rome, Italy

${ }^{4}$ Dipartimento di Fisica, Università di Siena and INFN Pisa, I-53100 Siena, Italy

5 Technische Universität Dortmund, D-44221 Dortmund, Germany

${ }^{6}$ Max-Planck-Institut für Physik, D-80805 München, Germany; jogler@ @lac.stanford.edu

${ }^{7}$ Dipartimento di Fisica, Università di Padova and INFN, I-35131 Padova, Italy

${ }^{8}$ Instituto de Astrofísica de Canarias, E-38200 La Laguna, Tenerife, Spain

${ }^{9}$ Department of Astrophysics, University of Łódź, PL-90236 Lodz, Poland

${ }^{10}$ Departamento de Astrofísica, Universidad de La Laguna, E-38206 La Laguna, Spain

${ }^{11}$ Deutsches Elektronen-Synchrotron (DESY), D-15738 Zeuthen, Germany

${ }^{12}$ ETH Zurich, CH-8093 Zurich, Switzerland

${ }^{13}$ Facultat de Fisica, Universitat de Barcelona (ICC/IEEC), E-08028 Barcelona, Spain; pmunar@am.ub.es

${ }^{14}$ Fakultät für Physik und Astronomie, Universität Würzburg, D-97074 Würzburg, Germany

${ }^{15}$ Dipartimento di Fisica Sperimentale, Università di Udine and INFN Trieste, I-33100 Udine, Italy

${ }^{16}$ Institut de Ciències de l'Espai (IEEC-CSIC), E-08193 Bellaterra, Spain

${ }^{17}$ Instituto de Astrofísica de Andalucía (CSIC), E-18080 Granada, Spain

${ }^{18}$ Croatian MAGIC Consortium, Rudjer Boskovic Institute, University of Rijeka and University of Split, HR-10000 Zagreb, Croatia

${ }^{19}$ Facultat de Fisica, Universitat Autònoma de Barcelona, E-08193 Bellaterra, Spain

${ }^{20}$ Tuorla Observatory, University of Turku, FI-21500 Piikkiö, Finland

${ }^{21}$ Institute for Nuclear Research and Nuclear Energy, BG-1784 Sofia, Bulgaria

22 Japanese MAGIC Consortium, Division of Physics and Astronomy, Kyoto University, Japan

${ }^{23}$ INAF/Osservatorio Astronomico and INFN, I-34143 Trieste, Italy

${ }^{24}$ Dipartimento di Fisica e Matematica, Università dell'Insubria, Como, I-22100 Como, Italy

${ }^{25}$ Dipartimento di Fisica, Università di Pisa and INFN Pisa, I-56126 Pisa, Italy

${ }^{26}$ ICREA, E-08010 Barcelona, Spain

Received 2012 March 5; accepted 2012 May 30; published 2012 July 5

\section{ABSTRACT}

The very high energy (VHE) $\gamma$-ray source HESS J0632+057 has recently been confirmed to be a $\gamma$-ray binary. The optical counterpart is the Be star MWC 148, and a compact object of unknown nature orbits it every 321 days with a high eccentricity of $\sim 0.8$. We monitored HESS J0632+057 with the stereoscopic MAGIC telescopes from 2010 October to 2011 March and detected significant VHE $\gamma$-ray emission during 2011 February, when the system exhibited an X-ray outburst. We find no $\gamma$-ray signal in the other observation periods when the system did not show increased X-ray flux. Thus, HESS J0632+057 exhibits $\gamma$-ray variability on timescales of the order of one to two months possibly linked to the X-ray outburst that takes place about 100 days after the periastron passage. Furthermore, our measurements provide for the first time the $\gamma$-ray spectrum down to about $140 \mathrm{GeV}$ and indicate no turnover of the spectrum at low energies. We compare the properties of HESS J0632+057 with 
the similar $\gamma$-ray binary LS I $+61^{\circ} 303$ and discuss the possible origin of the multi-wavelength emission of the source.

Key words: binaries: general - gamma rays: general - stars: individual (MWC 148) - X-rays: binaries - X-rays: individual (HESS J0632+057)

Online-only material: color figures

\section{INTRODUCTION}

The newest generation of Imaging Atmospheric Cherenkov Telescopes (IACTs) such as MAGIC, HESS, and VERITAS, could establish a new source class, the $\gamma$-ray binaries. Among the few known objects of this class, LS I +61 ${ }^{\circ} 303$, LS 5039, and PSR B1259-63 are regularly detected at very high energy (VHE; $E>100 \mathrm{GeV}$ ) $\gamma$-rays. All of these three systems show variable or even periodic VHE $\gamma$-ray emission and are spatially unresolvable by the current generation of IACTs (Aharonian et al. 2005, 2006; Albert et al. 2006, 2009).

HESS J0632+057 was discovered as an unidentified pointlike VHE $\gamma$-ray source but was considered to be a $\gamma$-ray binary candidate because of its spatial coincidence with the Be star MWC 148 (Aharonian et al. 2007; Hinton et al. 2009). The system was not detected in VHE $\gamma$-rays by VERITAS in an observation campaign from 2006 to 2009 (Acciari et al. 2009). These sparsely sampled measurements yielded flux upper limits (above $1 \mathrm{TeV}$ ) significantly below the previous detections, thus suggesting that HESS J0632+057 was variable in VHE $\gamma$-rays. Since all variable galactic VHE $\gamma$-ray sources known to date are associated with binary systems, ${ }^{32}$ HESS J0632+057 was a very good binary candidate. The here in detail presented VHE $\gamma$-ray detection simultaneously to the X-ray outburst in 2011 February was very recently announced by MAGIC and VERITAS (Ong 2011; Mariotti 2011; Jogler et al. 2011; Maier et al. 2011).

Measurements in soft X-rays with XMM-Newton detected an X-ray source (XMMU J063259.3+054801) at the position of MWC 148 (Hinton et al. 2009). The X-ray emission is well described by a hard power-law spectrum with energy spectral index $\Gamma=1.26 \pm 0.04$, consistent with emission of synchrotron radiation from VHE electrons, although a multitemperature spectral model can also reasonably describe the data. Furthermore, the X-ray source showed a variable flux, without changing the spectral shape. A similar behavior is seen, e.g., in the $\gamma$-ray binary LS I $+61^{\circ} 303$ (e.g., Anderhub et al. 2009). From 2009 January to 2009 May extensive X-ray observations with Swift/XRT found the source but at a different flux level and with a softer spectral index (Falcone et al. 2010). Recently published Swift/XRT observations from 2009 to 2011, display outbursts in the X-ray light curve from HESS J0632+057 with a periodicity of $P=321 \pm 5$ days (Bongiorno et al. 2011). These measurements also provided evidence for hardness ratio

\footnotetext{
${ }^{27}$ Now at Ecole polytechnique fédérale de Lausanne (EPFL), Lausanne, Switzerland.

${ }^{28}$ Now at Centro de Investigaciones Energéticas, Medioambientales y Tecnológicas (CIEMAT), Madrid, Spain.

29 Supported by INFN Padova.

${ }^{30}$ Now at KIPAC, SLAC National Accelerator Laboratory, USA.

${ }^{31}$ Now at Finnish Centre for Astronomy with ESO (FINCA), University of Turku, Finland.

${ }^{32}$ The Crab Nebula is variable at GeV energies, but no confirmed $\mathrm{TeV}$ variability has been measured up to now and thus it is not counted among the variable VHE $\gamma$-ray sources.
}

changes with orbital phase. The periodic X-ray emission is a strong evidence for HESS J0632+057 being a $\gamma$-ray binary. Chandra high time resolution X-ray measurements during the 2011 February X-ray outburst have been used to search for pulsed X-ray emission, but none was detected. Thus, the nature of the compact companion of MWC 148 remains unknown (Rea \& Torres 2011).

The search for a radio counterpart of HESS J0632+057 started in 2008 with the Very Large Array and the Giant Metrewave Radio Telescope at 5 and $1.28 \mathrm{GHz}$, respectively. These measurements exhibited an unresolved radio source within the position uncertainties of the VHE $\gamma$-ray source and the Be star MWC 148 (Skilton et al. 2009). A flux increase in the $5 \mathrm{GHz}$ band from $0.19 \pm 0.04$ to $0.41 \pm 0.04 \mathrm{mJy}$ showed the variability of the source on timescales of at least one month. The radio data were well described by a power-law spectrum with energy spectral index $\alpha=0.6 \pm 0.2$ using non-simultaneous data from $1.28 \mathrm{GHz}$ and $5 \mathrm{GHz}$. No extended structures beyond the 2 arcsec resolution were detected. During the 2011 February X-ray outburst very high resolution European Very Long Baseline Interferometry Network observations revealed a point-like source coincident with the Be star MWC 148 within uncertainties, which evolved into an extended source with a projected size of about $75 \mathrm{AU}$ (assuming a $1.5 \mathrm{kpc}$ distance), 30 days later (Moldón et al. 2011). The peak of the emission was displaced 21 AU between runs, which is bigger than the orbit size (semimajor axis $\sim 2.4 \mathrm{AU}$ ). The brightness temperature of the source was above $2 \times 10^{6} \mathrm{~K}$ hinting to a non-thermal origin of the particles producing the radio emission. The morphology, size, and displacement on AU scales were similar to those found in the other gamma-ray binaries, supporting a similar nature for HESS J0632+057 (Moldón et al. 2011). Further highresolution measurements will be needed to understand possible morphological changes in the radio structures along with the orbital phase.

Optical radial velocity measurements on MWC 148 yielded no significant variations at that time and simulations yielded a lower limit on the possible period of the system of $P>100$ days compatible with the period found later in X-rays (Aragona et al. 2010). Finally, radial velocity measurements with the Liverpool telescope obtained from 2008 to 2011 have proven the binary nature of HESS J0632+057/MWC 148. Fixing the orbital period to 321 days as obtained from the X-ray measurements by Bongiorno et al. (2011), these measurements provide for the first time the orbital parameters of the binary system. The compact object orbits MWC 148 on a highly eccentric ( $e=0.83 \pm 0.08$ ) orbit where the periastron passage occurs at phase $\phi_{\text {per }}=0.967 \pm 0.008$ using $T_{0}=$ MJD54857.0 (Casares et al. 2012).

In this Letter, we present the VHE $\gamma$-ray measurements of HESS J0632+057 by MAGIC from 2010 October to 2011 March. In particular we detect VHE $\gamma$-rays only during an $\mathrm{X}$-ray outburst in 2011 February and measure for the first time the spectrum down to $140 \mathrm{GeV}$. 


\section{OBSERVATIONS}

The observations of HESS J0632+057 were performed using the MAGIC telescopes on the Canary island of La Palma $(28.75 \mathrm{~N}, 17.86 \mathrm{~W}, 2225 \mathrm{~m}$ above sea level), from where HESS J0632+057 is observable at zenith angles above $22^{\circ}$. The MAGIC stereo system consists of two imaging air Cherenkov telescopes, each with a $17 \mathrm{~m}$ diameter reflector. Each telescope features a pixelized photomultiplier tube camera with a field of view of about 3.5. The observations were carried out in stereo mode, meaning that only shower images which simultaneously trigger both telescopes are recorded. The stereoscopic observation mode provides a sensitivity so that a $5 \sigma$ signal above $300 \mathrm{GeV}$ is detected from a source which exhibits $0.8 \%$ of the Crab Nebula flux in $50 \mathrm{hr}$ effective time. The angular resolution is better than 0.07 above several hundred $\mathrm{GeV}$ and the energy resolution is $16 \%$. Further details on the design and performance of the MAGIC stereo system can be found in Aleksić et al. (2012a).

We observed HESS J0632+057 between 2010 October and 2011 March for a total of $10.6 \mathrm{hr}$. All observations were carried out under moonlight conditions and at zenith angles from $22^{\circ}$ to $50^{\circ}$. The source was observed for several nights in each month and each of these observation sets are separated by about 20 days. This strategy maximizes the possibility of detecting emission from HESS J0632+057 in case of a long orbital period and with the system being active only during a short period of its orbit, i.e., one observation cycle. Due to bad weather no data were recorded in 2010 November and 2011 January.

\section{DATA ANALYSIS}

The data analysis was performed with the standard MAGIC analysis and reconstruction software (MARS). Events that triggered both telescopes were recorded and further processed. The recorded shower images were calibrated, cleaned, and used to calculate image parameters individually for each telescope. The energy of each event was then estimated using lookup tables generated by Monte Carlo (MC) simulated $\gamma$-ray events. In another step, further parameters, e.g., the height of the shower maximum and the impact parameter from each telescope, were calculated. The gamma/hadron classifications and reconstructions of the incoming direction of the primary particles were performed using the random forest (RF) method (Albert et al. 2008). The RF calculates the probability for each event to be of hadronic origin and denotes this parameter as the hadronness of the event. The signal selection uses cuts in the hadronness and in the squared angular distance between the shower pointing direction and the source position $\left(\theta^{2}\right)$. The energy-dependent cut values were determined by optimizing them on a sample of events recorded from the Crab Nebula under the same zenith angle range and similar epochs to HESS J0632+057 data. For the energy spectrum and flux, the effective detector area was estimated by applying the same cuts used on the data sample to a sample of MC-simulated $\gamma$-rays. Finally, the spectrum was unfolded in energy, accounting for the energy resolution and possible energy reconstruction bias (Albert et al. 2007).

The cuts used for producing the $\theta^{2}$ plot for the detection were optimized on a Crab Nebula data sample to yield the best sensitivity and have a higher energy threshold compared to the cuts used to produce the spectrum. For the light curve and integral flux calculations we chose a conservative energy threshold of $E_{\text {th }}=200 \mathrm{GeV}$, while the spectrum shows reconstructed signals

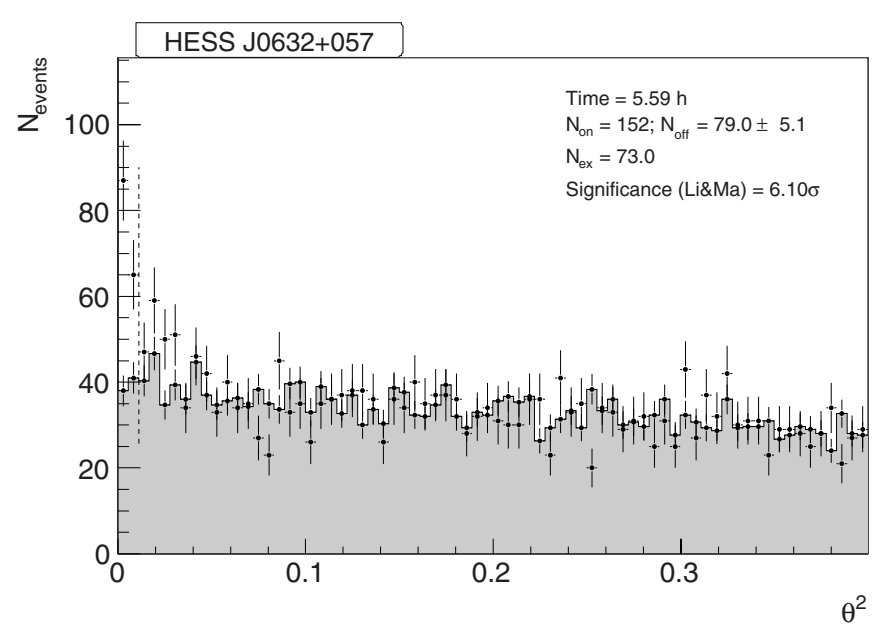

Figure 1. Squared angular distance between pointing direction of the shower and the source position $\left(\theta^{2}\right.$ plot) for the position of HESS J0632+057 (points) and the simultaneous determined background regions (gray shaded histogram) for the entire 2011 February MAGIC data set. The significance is calculated according to Li \& Ma (1983). $N_{\text {on }}$ is the number of events at the source position, $N_{\text {off }}$ is the number of background events, and $N_{\text {ex }}$ is the number of excess events $\left(N_{\text {ex }}=N_{\text {on }}-N_{\text {off }}\right)$.

down to $136 \mathrm{GeV}$. Note that the systematic uncertainties at the lowest energies dominate the total measurement uncertainties. Using a higher energy threshold, $200 \mathrm{GeV}$, guarantees smaller systematic uncertainties for the light curve and thus yields a better comparison to measurements from other instruments.

\section{RESULTS}

We detect VHE $\gamma$-ray emission from the HESS J0632+057 data set recorded in 2011 February, at an orbital phase separation of $\sim 0.3$ after periastron, with a significance of $6.1 \sigma$ in $5.6 \mathrm{hr}$ (see Figure 1). The VHE $\gamma$-ray source is not resolved by MAGIC and its extension must be smaller than the MAGIC point-spread function, whose Gaussian sigma is $0.09 \mathrm{deg}$ above $200 \mathrm{GeV}$. We obtain an integral flux of $F(E>200 \mathrm{GeV})=\left(8.8 \pm 1.7_{\text {stat }} \pm\right.$ $\left.2.1_{\text {syst }}\right) \times 10^{-12} \mathrm{~cm}^{-2} \mathrm{~s}^{-1}$ which corresponds to about $4 \%$ of the Crab Nebula flux. Previously reported detections (Aharonian et al. 2007) measured the flux only above $1 \mathrm{TeV}$ but agree well within the statistical uncertainties of our measurements when their spectrum is extended to our lower energy threshold. Thus, we conclude that during our observations, HESS J0632+057 exhibited similarly intense VHE emission to the previously detected active VHE $\gamma$-ray episodes (Aharonian et al. 2007).

The system was only detected in the 2011 February data during the X-ray outburst observed by Swift. No indication of significant emission was found in the data from 2010 October, 2010 December, or 2011 March. We denote these three months as the non-detection period (NDP). The integration time in the individual months of the NDP is, however, relatively short compared to the 2011 February and we combine the NDP to have the highest possible sensitivity for a baseline VHE flux. We obtain a flux upper limit for the NDP of $F(E>200 \mathrm{GeV})<$ $3.7 \times 10^{-12} \mathrm{~cm}^{-2} \mathrm{~s}^{-1}$ at the $95 \%$ confidence level following the method suggested by Rolke et al. (2005). Our flux upper limit excludes a baseline emission down to the level of $1.7 \%$ of the Crab Nebula flux. No individual night during the quiescent $\gamma$-ray state shows any indication of a signal.

We show in Figure 2 the obtained light curve above $200 \mathrm{GeV}$ of HESS J0632+057 for the nightly averages and provide the measurement details in Table 1. The VHE gamma-ray 


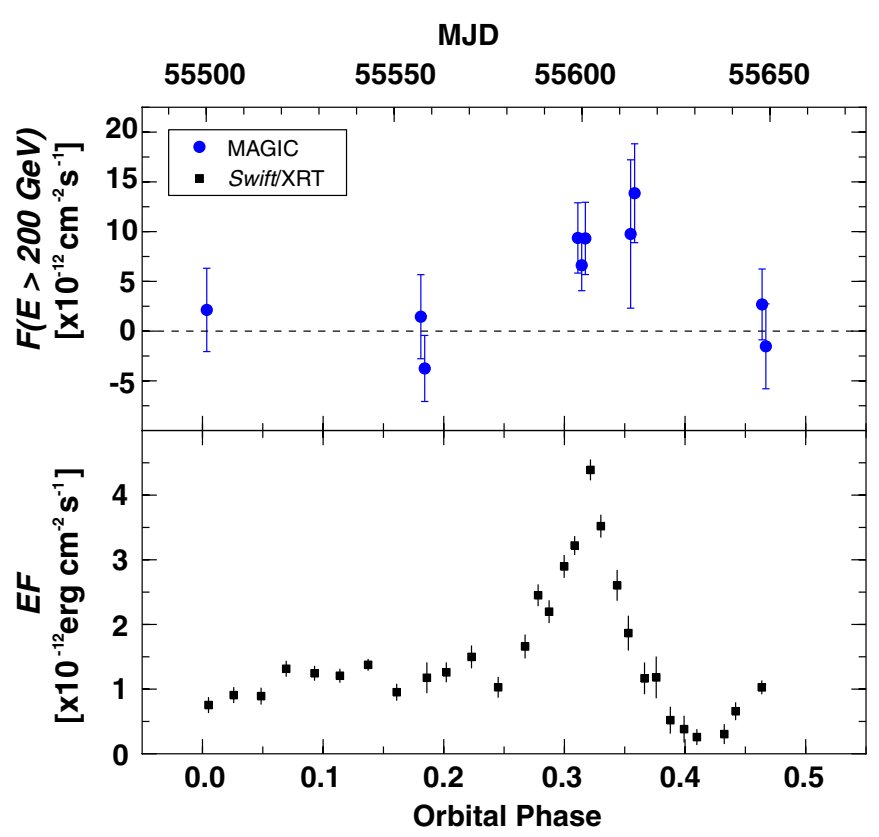

Figure 2. Light curve of HESS J0632+057 above $200 \mathrm{GeV}$ is shown in blue for the MAGIC observations (upper panel) and the Swift/XRT data between 2 and $10 \mathrm{keV}$ (lower panel) during the same orbital cycle as the MAGIC data in black. Significant emission in VHE is found only in 2011 February for the duration of about one month contemporary to the X-ray outburst. No variability in VHEs is seen during the active state. All error bars show the $1 \sigma$ statistical uncertainties.

(A color version of this figure is available in the online journal.)

Table 1

Observation Time, Orbital Phase, and Integral Flux (Above $200 \mathrm{GeV}$ )

\begin{tabular}{lcccr}
\hline \hline $\begin{array}{l}\text { Middle Time } \\
(\mathrm{MJD})\end{array}$ & $\begin{array}{c}\text { Obs. Time } \\
(\mathrm{min})\end{array}$ & Phase & $\begin{array}{c}\text { Significance } \\
\text { (Pre-trial) }\end{array}$ & $\begin{array}{c}\text { Flux } \\
10^{-12}\left(\mathrm{~cm}^{-2} \mathrm{~s}^{-1}\right)\end{array}$ \\
\hline 55500.141 & 45 & 0.00 & 2.1 & $4.2 \pm 0.5$ \\
55557.102 & 14 & 0.181 & 1.4 & $4.2 \pm 0.4$ \\
55558.134 & 88 & 0.184 & -1.1 & $3.8 \pm 3.3$ \\
55598.880 & 91 & 0.311 & 2.8 & $9.4 \pm 3.5$ \\
55599.903 & 133 & 0.314 & 2.7 & $6.6 \pm 2.6$ \\
55600.873 & 61 & 0.317 & 2.8 & $9.3 \pm 3.6$ \\
55612.920 & 14 & 0.355 & 1.5 & $9.8 \pm 7.5$ \\
55613.982 & 54 & 0.358 & 3.0 & $13.9 \pm 5.0$ \\
55647.899 & 112 & 0.463 & 0.8 & $2.7 \pm 3.6$ \\
55648.900 & 55 & 0.467 & -0.4 & $-1.5 \pm 4.3$ \\
\hline
\end{tabular}

Notes. All errors are statistical only we estimate an additional systematic uncertainty of about $40 \%$. The systematic uncertainty is only important when comparing between different experiments.

source exhibits variability timescales of about one month. Faster variability is possible but to detect it a denser sampling of the light curve is needed. However, no short timescale (days) variability is observed during the period of $\gamma$-ray activity in 2011 February. Under the assumption that there is no short-time variability in the time period when no VHE $\gamma$-ray emission is detected we conclude that the system shows flux variations of at least a factor of two between its quiescent and active state and that the active state must last between 20 and 80 days in the VHE regime.

A correlation of the VHE $\gamma$-ray emission with the 2011 February X-ray outburst is suggestive but cannot be proven statistically with our sparsely sampled light curve. More extensive observations in VHEs are needed for individual night correlation studies. Note that only in the time of high X-ray activity

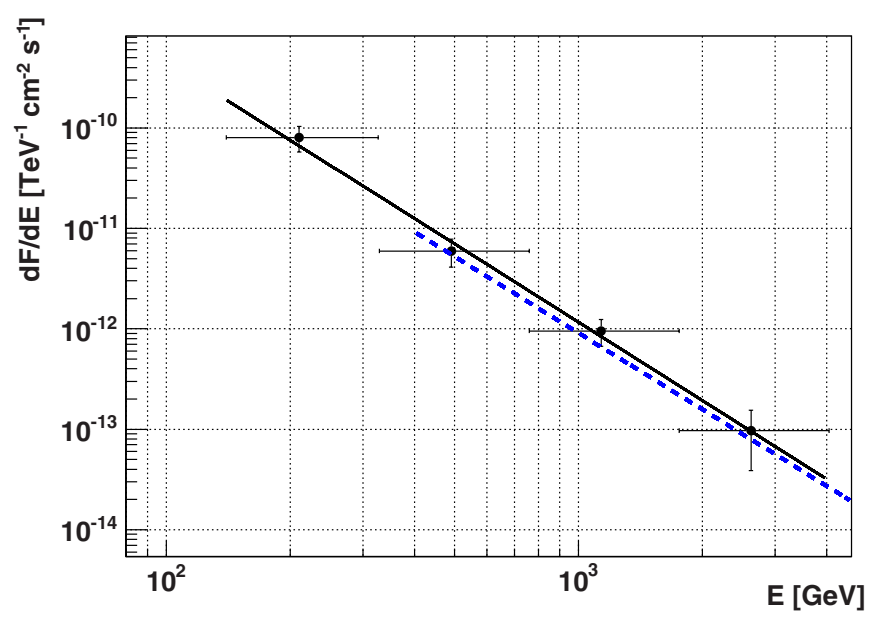

Figure 3. Differential energy spectrum of HESS J0632+057 between $136 \mathrm{GeV}$ and $4 \mathrm{TeV}$. The horizontal error bars represent the bin width whereas the vertical ones show the $1 \sigma$ statistical uncertainty. The spectrum is well described by the fitted simple power law with $\Gamma=-2.6 \pm 0.3_{\text {stat }} \pm 0.2_{\text {syst }}$ shown as the black line. The dashed (blue) line is the spectrum obtained by H.E.S.S. between 2004 March and 2006 March and is in good agreement with our measurement.

(A color version of this figure is available in the online journal.)

the system was detected by MAGIC. Whereas the X-ray light curve shows a clear peak shape for the outburst, the VHE light curve during the $\gamma$-ray activity is compatible with a constant flux. Whether this constant $\gamma$-ray flux is an artifact of the sparse sampling or a real characteristic of the outburst cannot be determined with these limited data. Note that a similar peak profile as in the X-ray outburst could be present in the VHE light curve.

We obtained a spectrum from the 2011 February data set and it is compatible with a simple power law (see Figure 3) with photon spectral index $2.6 \pm 0.3_{\text {stat }} \pm 0.2_{\text {syst }}$ and normalization $\left(1.2 \pm 0.3_{\text {stat }} \pm 0.2_{\text {syst }}\right) \times 10^{-12} \mathrm{TeV}^{-1} \mathrm{~cm}^{-2} \mathrm{~s}^{-1}$. The measured photon index is in very good agreement with the one previously published by H.E.S.S. $\left(\Gamma=2.53 \pm 0.26_{\text {stat }} \pm 0.2_{\text {syst }}\right)$, although their spectrum was obtained at energies above $400 \mathrm{GeV}$ (Aharonian et al. 2007). No indication of a turnover is found in the newly opened energy range by MAGIC.

\section{DISCUSSION}

The VHE gamma-ray data obtained during the periodic X-ray outburst of HESS J0632+057 that took place during 2011 February suggests that the VHE light curve shows similar outbursts like the X-ray light curve. The detection of VHE $\gamma$-ray emission only during the $\mathrm{X}$-ray outburst suggests a common origin although our data are too sparsely sampled to allow night to night correlation studies. The MAGIC detection of the source during the peak of the X-ray outburst yielded a similar flux level and energy spectrum as those obtained by HESS four years before (Aharonian et al. 2007). Similar spectral shape and flux levels indicate that the same processes might be at work during the $\gamma$-ray active states. In case of a periodic modulation with a period of about 321 days, such a behavior would be expected.

For the first time we could measure the spectrum of HESS J0632+057 between 136 and $400 \mathrm{GeV}$, and find no evidence for a spectral break or a deviation from a simple power law. This is a common feature found in other binaries which exhibit outbursts (e.g., LS I +61³03; Albert et al. 2009). Thus, the turnover in the spectrum must lie below the energy threshold of our observations. All currently known $\gamma$-ray binaries show their 
maximum emission in the high $\mathrm{MeV}$ to $\mathrm{GeV}$ energy range. This might be the case for HESS J0632+057 although it has not yet been detected by Fermi/LAT. The integration time required to detect HESS J0632+057 with Fermi/LAT will depend strongly on the source's duty cycle and the spectral properties at MeV to $\mathrm{GeV}$ energies. Assuming the shortest duty cycle compatible with our measurements (20 days) and taking the publicly available Fermi/LAT integral sensitivity curve ${ }^{33}$ we find that there must be a spectral brake between $140 \mathrm{GeV}$ and about $10 \mathrm{GeV}$. However, we note that the Fermi/LAT sensitivity curve is only available for extragalactic background and we scaled it by a factor of 10 to account for the higher galactic background. Since the galactic background varies depending on the region inside the galaxy, the Fermi/LAT sensitivity might be even lower at the position of HESS J0632+057. In case of a 100 times worse sensitivity compared to the extragalactic case we still find that the system should be detectable below $1 \mathrm{GeV}$ by Fermi/LAT.

In several recent publications, the similarity between HESS J0632+057 and LS I +61 303 was stressed based on their similar multiwavelength emission (e.g., Hinton et al. 2009; Skilton et al. 2009). We note that there might be some differences in the VHE emission. LS I $+61^{\circ} 303$ shows complex VHE behavior such as variability on timescales as short as one day (e.g., Anderhub et al. 2009), and different VHE $\gamma$-ray flux states (Acciari et al. 2011; Aleksić et al. 2012b). Revealing similar behavior in HESS J0632+057, requires higher sensitivity and better temporal sampled data than available to date and thus the degree of similarity between LS I $+61^{\circ} 303$ and HESS J0632+057 might be smaller compared to the contemporary view. Interestingly, the orbital phase lag for the detected VHE $\gamma$-ray emission in HESS J0632+057 is quite close to that in LS $\mathrm{I}+61^{\circ} 303$, about 0.3 after periastron passage ( $\mathrm{Li}$ et al. 2011; Casares et al. 2012). Similar processes might produce both the $\mathrm{X}$-ray and the VHE $\gamma$-ray radiation. However, different spatialand timescales of the emitter, given the wider and more eccentric orbit in HESS J0632+057, and different star-emitter-observer geometries (important in the leptonic scenario), make any direct comparison difficult. A proper characterization of the radiation and magnetic fields, and of possible adiabatic losses, is required. Nevertheless, one can check, under the light of the new data (see Hinton et al. 2009 for earlier attempts), whether a leptonic model can still describe the X-ray and VHE emission of HESS $\mathrm{J} 0632+057$, as it is the case in LS I +61 303 (Anderhub et al. 2009).

We have assumed an homogeneous (one-zone) emitter located at the compact object at orbital phase $\sim 0.3$, adopting system information provided in Casares et al. (2012). The free parameters are the magnetic field $(B)$, the electron acceleration rate $(\dot{E})$, the electron injection spectrum $(Q)$, and the adiabatic loss timescale $\left(t_{\mathrm{ad}}\right)$. All these quantities have been fixed through visual comparison with the data. We have taken a magnetic to stellar photon energy density ratio of $4 \times 10^{-4}, \dot{E}=0.2 q_{e} B c$, $Q \propto E^{-2}$, and $t_{\mathrm{ad}}=3 \times 10^{4} \mathrm{~s}$, to compute the radiation through synchrotron emission and inverse Compton (IC) scattering off stellar photons. The IC interaction geometry has been adopted at the orbital phase 0.3 , at which gamma-ray absorption is negligible. The computed X-ray and VHE spectral energy distributions are shown in Figure 4, together with the MAGIC and averaged Swift/XRT data taken during 2011 February. This simple model provides already a very reasonable description of the data, deviating only $\sim 2 \sigma$ from the lowest energy point derived by MAGIC

\footnotetext{
33 Available at: http://fermi.gsfc.nasa.gov/ssc/data/analysis/documentation/ Cicerone/Cicerone_LAT_IRFs/LAT_sensitivity.html
}

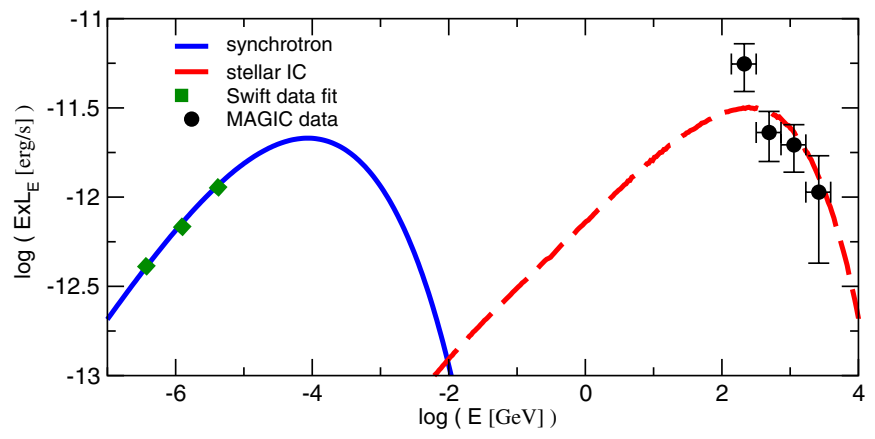

Figure 4. Spectral energy distribution for HESS J0632+057 as measured by MAGIC (full points) and Swift/XRT (thick dotted line) during the 2011 February outburst. We also show the modeling in a leptonic scenario where the X-ray and the VHE radiation is produced by synchrotron emission (solid line) and IC scattering off stellar photons (dashed line), respectively (see the text for details). (A color version of this figure is available in the online journal.)

(related to the Thomson-to-Klein-Nishina IC transition). Note however that a hadronic emission origin, although it requires an unrealistic magnetic field to explain the hard X-ray spectrum, cannot be discarded because of an X-ray luminosity in HESS J0632+057 slightly lower than at VHE (unlike LS I +61 303 ; see Anderhub et al. 2009).

To properly distinguish between the hadronic and leptonic pictures, and also model in detail the emitter, a better sampling of the light curve, and the X-ray/VHE spectral index correlation, are needed at the relevant orbital phases.

We thank the Instituto de Astrofísica de Canarias for the excellent working conditions at the Observatorio del Roque de los Muchachos in La Palma. The support of the German BMBF and MPG, the Italian INFN, the Swiss National Fund SNF, and the Spanish MICINN is gratefully acknowledged. This work was also supported by the Marie Curie program, by the CPAN CSD2007-00042 and MultiDark CSD2009-00064 projects of the Spanish Consolider-Ingenio 2010 programme, by grant DO02-353 of the Bulgarian NSF, by grant 127740 of the Academy of Finland, by the YIP of the Helmholtz Gemeinschaft, by the DFG Cluster of Excellence "Origin and Structure of the Universe," and by the Polish MNiSzW Grant N N203 390834.

\section{Facility: MAGIC}

\section{REFERENCES}

Acciari, V. A., Aliu, E., Arlen, T., et al. 2009, ApJ, 698, L94

Acciari, V. A., Aliu, E., Arlen, T., et al. 2011, ApJ, 738, 3

Aharonian, F., Akhperjanian, A. G., Bazer-Bachi, A. R., et al. 2006, A\&A, 460, 743

Aharonian, F. A., Akhperjanian, A. G., Aye, K.-M., et al. 2005, A\&A, 442, 1

Aharonian, F. A., Akhperjanian, A. G., Bazer-Bachi, A. R., et al. 2007, A\&A, 469, L1

Albert, J., Aliu, E., Anderhub, H., et al. 2006, Science, 312, 1771

Albert, J., Aliu, E., Anderhub, H., et al. 2007, Nucl. Instrum. Methods Phys. Res. A, 583, 494

Albert, J., Aliu, E., Anderhub, H., et al. 2008, Nucl. Instrum. Methods Phys. Res. A, 588, 424

Albert, J., Aliu, E., Anderhub, H., et al. 2009, ApJ, 693, 303

Aleksić, J., Alvarez, E. A., Antonelli, L. A., et al. 2012a, Astropart. Phys., 35, 435

Aleksić, J., Alvarez, E. A., Antonelli, L. A., et al. 2012b, ApJ, 746, 80

Anderhub, H., Antonelli, L. A., Antoranz, P., et al. 2009, ApJ, 706, L27 Aragona, C., McSwain, M. V., \& De Becker, M. 2010, ApJ, 724, 306

Bongiorno, S. D., Falcone, A. D., Stroh, M., et al. 2011, ApJ, 737, L11

Casares, J., Ribó, M., Ribas, I., et al. 2012, MNRAS, 421, 1103 
Falcone, A. D., Grube, J., Hinton, J., et al. 2010, ApJ, 708, L52

Hinton, J. A., Skilton, J. L., Funk, S., et al. 2009, ApJ, 690, L101

Jogler, T., Munar-Adrover, P., Ribó, M., \& Paredes, J. M., for the MAGIC Collaboration 2011, arXiv:1110.1682

Li, J., Torres, D. F., Zhang, S., et al. 2011, ApJ, 733, 89

Li, T.-P., \& Ma, Y.-Q. 1983, ApJ, 272, 317

Maier, G., for the VERITAS Collaboration, \& Skilton, J., for the HESS Collaboration 2011, arXiv:1111.2155
Mariotti, M. 2011, ATel, 3161, 1

Moldón, J., Ribó, M., \& Paredes, J. M. 2011, A\&A, 533, L7

Ong, R. A. 2011, ATel, 3153, 1

Rea, N., \& Torres, D. F. 2011, ApJ, 737, L12

Rolke, W., Lopez, A., \& Conrad, J. 2005, Nucl. Instrum. Methods Phys. Res. A, 551, 493

Skilton, J. L., Pandey-Pommier, M., Hinton, J. A., et al. 2009, MNRAS, 399, 317 